\section{High-sensitivity C-reactive protein/ albumin ratio as a predictor of in- hospital mortality in older adults admitted to the emergency department}

\author{
Jaehun Oh, Soo Hyun Kim, Kyu Nam Park, Sang Hoon Oh, \\ Young Min Kim, Han Joon Kim, Chun Song Youn \\ Department of Emergency Medicine, The Catholic University of Korea College of Medicine, Seoul, Korea
}

Objective The objective of this study was to test the hypothesis that an elevated high-sensitivity C-reactive protein (hs-CRP)/albumin ratio at admission increases the risk of mortality in older patients admitted to the hospital via the emergency department (ED).

Methods We performed a retrospective analysis of patients admitted to the ED with any medical problem between May 2013 and October 2013 who were older than 65 years. The hs-CRP and albumin levels were measured at the time of admission to the ED. The primary outcome was all-cause in-hospital mortality. Multivariate logistic analysis was performed.

Results A total of 811 patients were finally included in this study. The mean age was $76 \pm 7$ years, and 438 subjects (54\%) were male. The in-hospital mortality rate was $9.0 \%$ (73 patients). The hs-CRP/albumin ratio was higher in nonsurvivors than in survivors ( $34.2 \pm 37.6$ vs. $16.2 \pm 25.5$, $\mathrm{P}<0.001$ ). Multivariate logistic analysis showed that the hs-CRP/albumin ratio was associated with all-cause in-hospital mortality after adjusting for other confounding factors (odds ratio, $1.011 ; 95 \%$ confidence interval $[\mathrm{Cl}], 1.003$ to 1.020 ). The prognostic value of the hs-CRP/albumin ratio for predicting mortality (area under the curve, $0.728 ; 95 \% \mathrm{Cl}, 0.696$ to 0.758 ) was greater than that of hs-CRP alone (area under the curve, $0.706 ; 95 \% \mathrm{Cl}, 0.674$ to $0.738 ; \mathrm{P}<0.001$ ).

Conclusion The hs-CRP/albumin ratio at admission to the ED is associated with all-cause inhospital mortality among patients older than 65 years. The hs-CRP/albumin ratio may serve as a surrogate marker of disease severity.

Keywords Mortality; Aged; C-reactive protein; Albumins

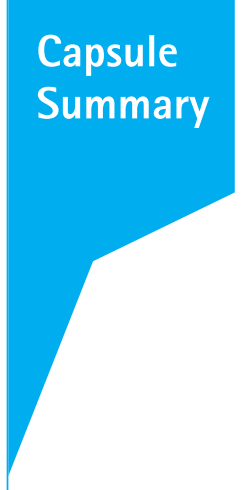

What is already known

The high-sensitivity C-reactive protein (hs-CRP)/albumin ratio has recently been tested as a prognostic marker for mortality in sepsis and acute kidney injury. The hs-CRP/albumin ratio has more value than either test alone for predicting prognoses in various clinical settings.

\section{What is new in the current study}

The hs-CRP/albumin ratio at admission to the emergency department is associated with all-cause in-hospital mortality among patients older than 65 years. A high hs-CRP/albumin ratio at initial presentation to the emergency department among older patients may provide evidence to aid the decision-making process regarding admission to the hospital regardless of etiology.
eISSN: 2383-4625

Received: 21 November 2016

Revised: 15 December 2016

Accepted: 27 December 2016

Correspondence to: Chun Song Youn Department of Emergency Medicine, Seoul St. Mary's Hospital, 222 Banpodaero, Seocho-gu, Seoul 06591, Korea E-mail:ycs1005@catholic.ac.kr

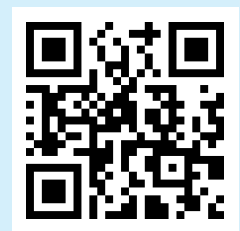

How to cite this article:

Oh J, Kim SH, Park KN, Oh SH, Kim YM, Kim HJ, Youn CS. High-sensitivity C-reactive protein/albumin ratio as a predictor of inhospital mortality in older adults admitted to the emergency department. Clin Exp Emerg Med 2017;4(1):19-24.

This is an Open Access article distributed under the terms of the Creative Commons Attribution Non-Commercial License (http:// creativecommons.org/licenses/by-nc/4.0/). 


\section{INTRODUCTION}

The global population is aging worldwide, and this has been a major issue in healthcare. ${ }^{1}$ The aging of the population in Korea is projected to be the fastest in the Organization for Economic Cooperation and Development countries. ${ }^{2}$ Older adults visit the emergency department (ED) more frequently than younger adults. ${ }^{3,4}$ Accurate diagnosis and treatment of older adults is more complex because they tend to have atypical signs and symptoms with multiple comorbidities. ${ }^{5,6}$ Moreover, they are at a high risk of hospitalization, intensive care unit admission, and death. ${ }^{7}$ However, the association between risk factors and in-hospital mortality in older adults admitted to the ED has rarely been investigated.

High-sensitivity C-reactive protein (hs-CRP) is an acute-phase reactant and a marker of acute and chronic inflammation. ${ }^{8}$ The hs-CRP level is a marker for predicting the risk or prognosis of various diseases such as coronary artery disease (CAD), ischemic stroke, sepsis, and cancer. ${ }^{9-14}$ Albumin is an indicator of malnutrition, and hypoalbuminemia is a prognostic factor in hospitalized elderly patients. ${ }^{15,16}$ For this reason, the combination of these two parameters, namely the ratio of hs-CRP to albumin, may have more value than either of them individually for predicting prognoses in various clinical settings, and this ratio has recently been tested as a prognostic marker for mortality in sepsis and acute kidney injury. ${ }^{17,18}$ Moreover, older patients are susceptible to infectious disease, and nutritional status is an important prognostic factor in various geriatric clinical settings.

We tested the hypothesis that an elevated hs-CRP/albumin ratio at admission increases the risk of mortality in older patients admitted to the hospital via the ED. We assessed the association between the hs-CRP/albumin ratio at admission and in-hospital mortality in older patients admitted to the hospital via the ED.

\section{METHODS}

\section{Study design and setting}

This retrospective study was conducted in the Department of Emergency Medicine of Seoul St. Mary's Hospital, a tertiary educational hospital. This study was approved by our institutional review board, and a requirement for consent was waived owing to its retrospective design. Patients were included if they were older than 65 years and admitted to our hospital via the ED with any medical problem between May 2013 and October 2013. Patients were excluded if they had a trauma-related injury or were discharged from the ED.

\section{Laboratory measurements}

Blood samples for a complete blood count and serum chemistry including serum albumin and hs-CRP levels were obtained when admitted to the ED as part of the routine testing in all adult patients with any medical problem. The hs-CRP level was measured using a turbidimetric immunoassay (Wako Chemicals, Osaka, Japan) on a Hitachi 7600 chemistry analyzer (Hitachi, Tokyo, Japan). The analytical sensitivity of the assay was $0.2 \mathrm{mg} / \mathrm{L}$, and the intraassay coefficient of variation was $<5 \%$.

\section{Data collection}

Demographic and clinical data were collected from electronic medical records. All comorbidities such as cancer, diabetes, hypertension, $C A D$, cerebrovascular accident, congestive heart failure, chronic kidney disease, and chronic pulmonary disease were also collected and defined according to the International Classification of Diseases, 10th revision. ${ }^{19}$ Laboratory parameters such as blood urea nitrogen, creatinine, hemoglobin, sodium, and CRP were also included. A Sequential Organ Failure Assessment (SOFA) score was calculated at the time of ED admission. The primary outcome was all-cause in-hospital mortality. Survival to hospital discharge was defined as discharge from the hospital alive to home or to another healthcare facility including a rehabilitation hospital.

\section{Statistical analysis}

Data are expressed as means \pm standard deviations for continuous variables and percentages for categorical variables. Student's ttest for continuous variables and Fisher's exact test and the $\chi^{2}$ test for categorical variables were used. To find the factors associated with in-hospital mortality, univariate analyses were performed first followed by multivariate logistic regression analysis. Variables with P-values $<0.05$ on univariate analysis were entered into the multivariate logistic regression model. To evaluate the association of the hs-CRP/albumin ratio with mortality outcomes, hs-CRP/albumin ratio values were divided into four categories using the following cutoff values: $<0.59,0.59-5.03,5.05-$ 25.07 , and $>25.07$. Odds ratios and $95 \%$ confidence intervals (Cls) were calculated with the lowest quartile as the reference. The hs-CRP/albumin ratio was also examined as a continuous variable. To determine the best cutoff values for the albumin and hs-CRP levels and hs-CRP/albumin ratio, a receiver operating characteristic curve was generated and Youden's index was calculated; sensitivities, specificities, positive and negative predictive values, and their 95\% Cls were also calculated. We determined predictive performance using receiver operating characteristic curves with logistic regression models to compare and assess for equality of the area under the curve using the DeLong test. Statistical 
analyses were performed using IBM SPSS ver. 20.0 (IBM Corp., Armonk, NY, USA) and MedCalc ver. 15.2.2 (MedCalc Software, Mariakerke, Belgium), and P-values $\leq 0.05$ were considered statistically significant.

\section{RESULTS}

\section{Characteristics of the subjects}

A total of 5,166 patients older than 65 years were admitted to our ED during the study period. Of those, 4,355 patients were excluded because they had a trauma-related injury $(n=621)$, were transferred from another hospital $(n=723)$, were dead on arrival $(n=129)$, or visited the ED for irrelevant medical treatment $(n=52)$ and did not admitted to our hospital $(n=2,830)$. The remaining 811 patients were finally included in this study. The mean age was $76 \pm 7$ years, and 438 patients (54\%) were male. Seventy-three patients (9.0\%) died during their hospital stay. Hypertension was the most common comorbidity. Men were more common in the nonsurvivor group. A history of cancer was more common among nonsurvivors, whereas hypertension was more common among survivors. Baseline characteristics and laboratory data are shown in Table 1.
Table 1. Baseline characteristics of patients with or without in-hospital mortality

\begin{tabular}{lccr}
\hline & $\begin{array}{c}\text { Survivors } \\
(\mathrm{n}=738)\end{array}$ & $\begin{array}{c}\text { Nonsurvivors } \\
(\mathrm{n}=73)\end{array}$ & P-value \\
\hline Age (yr) & $75.6 \pm 7.3$ & $75.0 \pm 7.6$ & 0.514 \\
Sex, male & $390(52.8)$ & $48(65.8)$ & 0.035 \\
Cancer & $213(28.9)$ & $46(63.0)$ & $<0.001$ \\
Diabetes mellitus & $257(34.8)$ & $18(24.7)$ & 0.080 \\
Hypertension & $473(64.1)$ & $33(45.2)$ & 0.001 \\
CAD & $124(16.8)$ & $5(6.8)$ & 0.027 \\
CVA & $103(14.0)$ & $6(8.2)$ & 0.170 \\
CHF & $35(4.7)$ & $5(6.8)$ & 0.428 \\
CKD & $77(10.4)$ & $8(11.0)$ & 0.889 \\
Lung disease & $134(18.2)$ & $17(23.3)$ & 0.283 \\
BUN (mg/dL) & $27.1 \pm 21.6$ & $34.3 \pm 20.8$ & 0.007 \\
Creatinine (mg/dL) & $1.5 \pm 1.9$ & $1.5 \pm 1.0$ & 0.930 \\
Hemoglobin (g/dL) & $12.2 \pm 2.5$ & $11.2 \pm 2.3$ & 0.002 \\
Albumin (g/dL) & $3.7 \pm 0.6$ & $3.0 \pm 0.6$ & $<0.001$ \\
Sodium (mEq/L) & $137.2 \pm 6.1$ & $135.5 \pm 7.5$ & 0.024 \\
hs-CRP (mg/dL) & $5.2 \pm 7.7$ & $9.1 \pm 8.2$ & $<0.001$ \\
SOFA score & $2.4 \pm 1.7$ & $4.4 \pm 2.8$ & $<0.001$ \\
hs-CRP/albumin ratio & $16.2 \pm 25.5$ & $34.2 \pm 37.6$ & $<0.001$ \\
\hline & & &
\end{tabular}

Values are presented as mean \pm standard deviation or number $(\%)$.

$C A D$, coronary artery disease; $C V A$, cerebrovascular disease; $C H F$, congestive heart failure; CKD, chronic kidney disease; BUN, blood urea nitrogen; hs-CRP, high-sensitivity C-reactive protein; SOFA, Sequential Organ Failure Assessment.

Table 2. ORs for all-cause in-hospital mortality events according to the hs-CRP/albumin ratio as a categorical variable

\begin{tabular}{|c|c|c|c|c|c|c|}
\hline \multirow{2}{*}{ Variable } & \multicolumn{3}{|c|}{ Univariate } & \multicolumn{3}{|c|}{ Multivariate } \\
\hline & $\mathrm{OR}$ & $95 \% \mathrm{Cl}$ & P-value & OR & $95 \% \mathrm{Cl}$ & P-value \\
\hline Age & 0.989 & $0.956-1.023$ & 0.514 & - & - & - \\
\hline Sex, male & 1.713 & $1.034-2.838$ & 0.036 & 1.631 & $0.922-2.885$ & 0.093 \\
\hline Cancer & 4.199 & $2.544-6.931$ & $<0.001$ & 3.153 & $1.754-5.668$ & $<0.001$ \\
\hline Diabetes mellitus & 0.613 & $0.352-1.065$ & 0.083 & - & - & - \\
\hline Hypertension & 0.462 & $0.285-0.751$ & 0.002 & 0.641 & $0.365-1.128$ & 0.123 \\
\hline CAD & 0.364 & $0.144-0.921$ & 0.033 & 0.487 & $0.178-1.330$ & 0.160 \\
\hline CVA & 0.552 & $0.233-1.306$ & 0.176 & - & - & - \\
\hline CHF & 1.477 & $0.560-3.894$ & 0.431 & - & - & - \\
\hline CKD & 1.057 & $0.489-2.285$ & 0.889 & - & - & - \\
\hline Lung disease & 1.368 & $0.771-2.430$ & 0.284 & - & - & - \\
\hline BUN (mg/dL) & 1.012 & $1.003-1.021$ & 0.009 & 1.003 & $0.990-1.017$ & 0.618 \\
\hline Creatinine (mg/dL) & 0.994 & $0.871-1.134$ & 0.930 & - & - & - \\
\hline Hemoglobin (g/dL) & 0.864 & $0.787-0.949$ & 0.002 & 0.972 & $0.866-1.091$ & 0.630 \\
\hline Albumin (g/dL) & 0.185 & $0.123-0.279$ & $<0.001$ & - & - & - \\
\hline Sodium (mEq/L) & 0.962 & $0.931-0.995$ & 0.025 & 1.000 & $0.957-1.044$ & 0.989 \\
\hline hs-CRP (mg/dL) & 1.049 & $1.024-1.076$ & $<0.001$ & - & - & - \\
\hline SOFA score & 1.488 & $1.338-1.655$ & $<0.001$ & 1.412 & $1.235-1.614$ & $<0.001$ \\
\hline \multicolumn{7}{|l|}{ hs-CRP/albumin ratio } \\
\hline Quartile 1 & REF & REF & REF & REF & REF & REF \\
\hline Quartile 2 & 2.393 & $0.610-9.388$ & 0.211 & 1.700 & $0.422-6.845$ & 0.456 \\
\hline Quartile 3 & 12.016 & $3.610-39.992$ & $<0.001$ & 5.599 & $1.622-19.328$ & 0.006 \\
\hline Quartile 4 & 12.476 & $3.754-41.459$ & $<0.001$ & 5.940 & $1.708-20.655$ & 0.005 \\
\hline
\end{tabular}

$\mathrm{OR}$, odds ratio; hs-CRP, high-sensitivity $\mathrm{C}$-reactive protein; $\mathrm{Cl}$, confidence interval; $\mathrm{CAD}$, coronary artery disease; $\mathrm{CVA}$, cerebrovascular disease; $\mathrm{CHF}$, congestive heart failure; CKD, chronic kidney disease; BUN, blood urea nitrogen; SOFA, Sequential Organ Failure Assessment; REF, reference. 
Table 3. ORs for all-cause in-hospital mortality events according to hs-CRP/albumin ratio as a continuous variable

\begin{tabular}{|c|c|c|c|c|c|c|}
\hline \multirow{2}{*}{ Variable } & \multicolumn{3}{|c|}{ Univariate } & \multicolumn{3}{|c|}{ Multivariate } \\
\hline & OR & $95 \% \mathrm{Cl}$ & P-value & OR & $95 \% \mathrm{Cl}$ & P-value \\
\hline Age & 0.989 & $0.956-1.023$ & 0.514 & - & - & - \\
\hline Sex, male & 1.713 & $1.034-2.838$ & 0.036 & 1.664 & $0.941-2.940$ & 0.080 \\
\hline Cancer & 4.199 & $2.544-6.931$ & $<0.001$ & 3.531 & $1.971-6.327$ & $<0.001$ \\
\hline Diabetes mellitus & 0.613 & $0.352-1.065$ & 0.083 & - & - & - \\
\hline Hypertension & 0.462 & $0.285-0.751$ & 0.002 & 0.575 & $0.329-1.005$ & 0.052 \\
\hline CAD & 0.364 & $0.144-0.921$ & 0.033 & 0.486 & $0.180-1.313$ & 0.155 \\
\hline CVA & 0.552 & $0.233-1.306$ & 0.176 & - & - & - \\
\hline CHF & 1.477 & $0.560-3.894$ & 0.431 & - & - & - \\
\hline CKD & 1.057 & $0.489-2.285$ & 0.889 & - & - & - \\
\hline Lung disease & 1.368 & $0.771-2.430$ & 0.284 & - & - & - \\
\hline BUN (mg/dL) & 1.012 & $1.003-1.021$ & 0.009 & 1.003 & $0.990-1.016$ & 0.616 \\
\hline Creatinine $(\mathrm{mg} / \mathrm{dL})$ & 0.994 & $0.871-1.134$ & 0.930 & - & - & - \\
\hline Hemoglobin (g/dL) & 0.864 & $0.787-0.949$ & 0.002 & 0.962 & $0.859-1.078$ & 0.506 \\
\hline Albumin (g/dL) & 0.185 & $0.123-0.279$ & $<0.001$ & - & - & - \\
\hline Sodium (mEq/L) & 0.962 & $0.931-0.995$ & 0.025 & 0.992 & $0.951-1.035$ & 0.710 \\
\hline hs-CRP (mg/dL) & 1.049 & $1.024-1.076$ & $<0.001$ & - & - & - \\
\hline SOFA score & 1.488 & $1.338-1.655$ & $<0.001$ & 1.430 & $1.254-1.631$ & $<0.001$ \\
\hline hs-CRP/albumin ratio & 1.017 & $1.010-1.024$ & $<0.001$ & 1.011 & $1.003-1.020$ & 0.005 \\
\hline
\end{tabular}

$\mathrm{OR}$, odds ratio; hs-CRP, high-sensitivity C-reactive protein; $\mathrm{Cl}$, confidence interval; $\mathrm{CAD}$, coronary artery disease; $\mathrm{CVA}$, cerebrovascular disease; $\mathrm{CHF}$, congestive heart failure CKD, chronic kidney disease; BUN, blood urea nitrogen; SOFA, Sequential Organ Failure Assessment.

Table 4. Sensitivity, specificity, positive predictive value, and negative predictive value of albumin, hs-CRP, and hs-CRP/albumin ratio

\begin{tabular}{lccrrr}
\hline & Cutoff & Sensitivity & Specificity & PPV & NPV \\
\hline Albumin $(\mathrm{g} / \mathrm{dL})$ & $\leq 3.4$ & $78.1(66.9-86.9)$ & $68.7(65.2-72.0)$ & $19.8(15.3-24.9)$ & $96.9(95.1-98.2)$ \\
hs-CRP $(\mathrm{mg} / \mathrm{dL})$ & $>1.58$ & $89.0(79.5-95.1)$ & $51.4(47.7-55.0)$ & $15.3(12.0-19.1)$ & $97.9(96.0-99.1)$ \\
hs-CRP/albumin ratio & $>6.44$ & $86.3(76.2-93.2)$ & $56.78(53.1-60.4)$ & $16.5(12.9-20.6)$ & $97.7(95.7-98.9)$ \\
\hline
\end{tabular}

Parenthesis is 95\% confidence interval.

hs-CRP, high-sensitivity C-reactive protein; PPV, positive predictive value; NPV, negative predictive value.

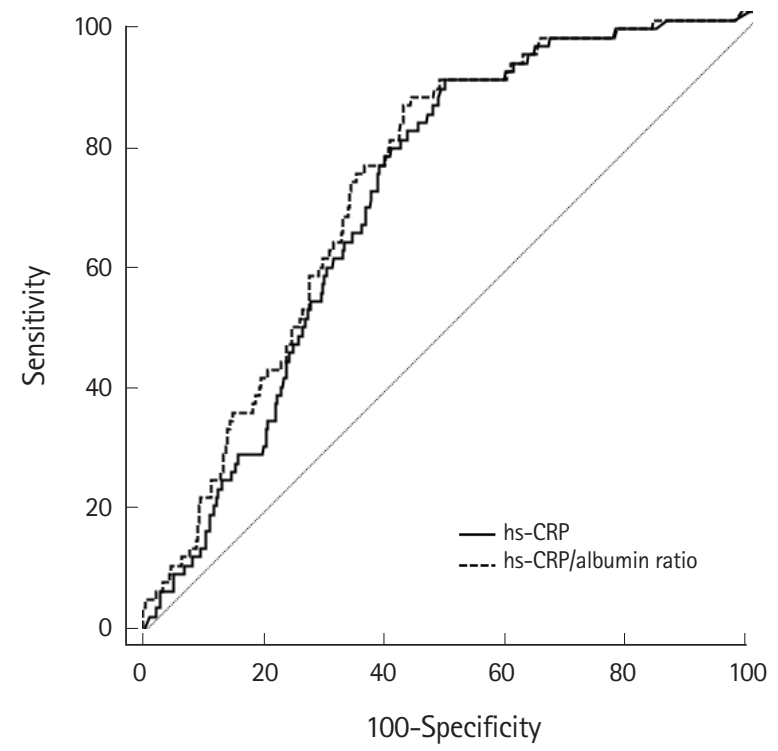

Fig. 1. Receiver operating characteristics curve of high-sensitivity C-reactive protein (hs-CRP) and hs-CRP/albumin ratio to predict in-hospital mortality. The area under the curve of the hs-CRP/albumin ratio and hsCPR is 0.728 (95\% confidence interval, 0.696 to 0.758$)$ and 0.706 (95\% confidence interval, 0.674 to 0.738$)$, respectively $(\mathrm{P}<0.001)$.

\section{Logistic regression analysis}

The hs-CRP/albumin ratio ranged from 0.04 to 179.47 (17.9 \pm 27.3$)$. The hs-CRP/albumin ratio was higher in nonsurvivors than in survivors $(34.2 \pm 37.6$ vs. $16.2 \pm 25.5, P<0.001)$. In the univariate analyses, male sex, history of cancer, hypertension, CAD, blood urea nitrogen, hemoglobin, albumin, sodium, hs-CRP, and SOFA values showed statistically significant associations with all-cause in-hospital mortality.

After adjusting for these variables, the hs-CRP/albumin ratio still showed an association with all-cause in-hospital mortality. Patients in the fourth quartile were 5.94 times more likely to die compared with those in the lowest quartile of the hs-CRP/albumin ratio (Table 2). When the hs-CRP/albumin ratio was examined as a continuous variable, it still showed an association with all-cause in-hospital mortality (odds ratio, 1.011; 95\% Cl, 1.003 to 1.020) (Table 3).

\section{Prognostic value of the hs-CRP/albumin ratio}

Table 4 shows the sensitivity, specificity, and positive and negative predictive values of the albumin and hs-CRP levels and the 
Table 5. Cause of death

\begin{tabular}{lc}
\hline Cause of death & Number \\
\hline Cancer & 29 \\
Sepsis & 20 \\
Respiratory failure & 16 \\
Heart failure & 4 \\
Neurologic disorder & 2 \\
Pancreatitis & 1 \\
Pulmonary thromboembolism & 1
\end{tabular}

hs-CRP/albumin ratio. The best cutoff value of the hs-CRP/albumin ratio is 6.44 , with $86.3 \%$ sensitivity and $56.8 \%$ specificity. The area under the curve of the hs-CRP/albumin ratio $(0.728$; $95 \% \mathrm{Cl}, 0.696$ to 0.758 ) was greater than that of hs-CRP alone $(0.706 ; 95 \% \mathrm{Cl}, 0.674$ to $0.738 ; \mathrm{P}<0.001)$ (Fig. 1). Table 5 shows the cause of death of individual patients.

\section{DISCUSSION}

The main finding of this study was that the hs-CRP/albumin ratio at admission was an independent predictor of all-cause in-hospital mortality among patients older than 65 years. To the best of our knowledge, this is the first report describing the association between the hs-CRP/albumin ratio and in-hospital mortality among older patients. After adjusting for multiple confounding factors, the all-cause in-hospital mortality rate was increased by $1.1 \%$ for each whole-number increase in the hs-CRP/albumin ratio. Furthermore, the prognostic value of the hs-CRP/albumin ratio for predicting mortality was greater than that of hs-CRP alone. If a patient had a hs-CRP/albumin ratio of $>6.44$, the risk of inhospital death was $16.4 \%$. If a patient had a hs-CRP/albumin ratio of $<6.44$, the risk of survival hospital discharge was $97.7 \%$.

The increasing elderly population is a big social and health problem. ${ }^{1}$ Elderly patients visit the ED more frequently than young adults. Moreover, accurate diagnosis and treatment is more complex because they have multiple comorbidities, increasing the difficulty of predicting mortality. The association between risk factors and in-hospital mortality in older adults admitted to the ED has rarely been investigated. Therefore, a strategy based on the targeting of high-risk patients has been proposed. ${ }^{6}$ The results of this study may also assist in the identification of older patients at high risk of mortality. One interesting finding of our results is that the history of hypertension and CAD decreased the odds for in-hospital mortality in univariate logistic regression analysis. Further testing should be done to determine the association between a history of hypertension and CAD and in-hospital mortality among older patients.
Albumin levels are a sensitive index for nutritional status. Many factors influence serum levels of albumin such as kidney disease and the patient's state of hydration. Hypoalbuminemia is associated with higher mortality during hospital stays in older patients. ${ }^{15,16}$ However, it remains to be proven whether adequate correction of hypoalbuminemia can reduce mortality.

CRP is an acute-phase reactant and a nonspecific marker in the diagnosis of various conditions such as infectious diseases and autoimmune and rheumatologic disorders. The clinical usefulness of CRP has widened with the availability of high-sensitivity assays that can measure CRP levels as low as $0.1 \mathrm{mg} / \mathrm{L}$. Elevated levels of hs-CRP are associated with the prognosis of various diseases such as CAD, ischemic stroke, sepsis, and cancer. ${ }^{9-14}$

Both hs-CRP and albumin could be prognostic markers for outcomes in various clinical settings, but the combination of these markers may have more value than either one alone because this would include both inflammatory and nutritional information. ${ }^{18}$ The CRP/albumin ratio was associated with 90-day and 180-day mortality in patients with sepsis, ${ }_{1}^{17,20}$ and the CRP/albumin ratio had a higher predictive value than CRP alone. In our study, the hs-CRP/albumin ratio at ED admission was positively correlated with mortality among patients older than 65 years. Our study suggests that the hs-CRP/albumin ratio may serve as an early surrogate marker for mortality of the entire spectrum of acute disease in elderly patients and is not confined to any specific disease.

The prediction of in-hospital mortality or risk stratification among older patients admitted to the ED has rarely been investigated. Some authors have suggested scoring systems, but they have not been externally validated. ${ }^{21}$ Vital signs and level of consciousness, which have been used in other scoring systems, were not included in our study. However, we included a SOFA score that includes mean arterial pressure and the Glasgow Coma Scale. ${ }^{22}$ Therefore, this is not a limitation of our study.

There are some limitations in this study. The first limitation is that this study used a retrospective observational design at a single center, which raises the concern of potential unknown confounding factors and limits the generalizability of its results. Second, the primary outcome of this study was all-cause in-hospital mortality, and we could not identify the association between the hs-CRP/albumin ratio and cause-specific mortality because only 73 patients (9.0\%) died during their hospital stay. Third, the physicians were not blinded to the hs-CRP and albumin results because they are routinely reported in our hospital. This could be a potential source of bias and could affect the prognostic value of the hs-CRP/albumin ratio. However, the hs-CRP/albumin ratio was not used for any clinical decisions such as hospital admission, critical procedure, or discharge from our hospital. 
In conclusion, the hs-CRP/albumin ratio at admission to the ED was associated with all-cause in-hospital mortality among patients older than 65 years. The hs-CRP/albumin ratio may serve as a surrogate marker of disease severity. A high hs-CRP/albumin ratio at initial presentation to the ED among older patients may provide evidence to aid in the decision-making process regarding admission to the hospital regardless of etiology.

\section{CONFLICT OF INTEREST}

No potential conflict of interest relevant to this article was reported.

\section{REFERENCES}

1. Mion LC, Palmer RM, Anetzberger GJ, Meldon SW. Establishing a case-finding and referral system for at-risk older individuals in the emergency department setting: the SIGNET model. J Am Geriatr Soc 2001;49:1379-86.

2. Organization for Economic Cooperation and Development. OECD economic surveys: Korea. Paris: Organization for Economic Cooperation and Development; 2016.

3. Niska R, Bhuiya F, Xu J. National Hospital Ambulatory Medical Care Survey: 2007 emergency department summary. Natl Health Stat Report 2010;26:1-31.

4. Roberts DC, McKay MP, Shaffer A. Increasing rates of emergency department visits for elderly patients in the United States, 1993 to 2003. Ann Emerg Med 2008;51:769-74.

5. Aminzadeh F, Dalziel WB. Older adults in the emergency department: a systematic review of patterns of use, adverse outcomes, and effectiveness of interventions. Ann Emerg Med 2002;39:238-47.

6. Samaras N, Chevalley T, Samaras D, Gold G. Older patients in the emergency department: a review. Ann Emerg Med 2010; 56:261-9.

7. Strange GR, Chen EH. Use of emergency departments by elder patients: a five-year follow-up study. Acad Emerg Med 1998; 5:1157-62.

8. Danesh J, Wheeler JG, Hirschfield GM, et al. C-reactive protein and other circulating markers of inflammation in the prediction of coronary heart disease. N Engl J Med 2004;350:1387-97.

9. Emerging Risk Factors Collaboration, Kaptoge S, Di Angelan-

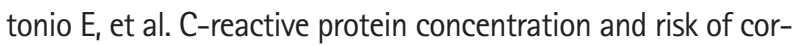
onary heart disease, stroke, and mortality: an individual participant meta-analysis. Lancet 2010;375:132-40.
10. Oemrawsingh RM, Cheng JM, Akkerhuis KM, et al. High-sensitivity C-reactive protein predicts 10 -year cardiovascular outcome after percutaneous coronary intervention. Eurolntervention 2016;12:345-51.

11. Matsuo R, Ago T, Hata J, et al. Plasma C-reactive protein and clinical outcomes after acute ischemic stroke: a prospective observational study. PLoS One 2016;11:e0156790.

12. Elkind MS, Luna JM, McClure LA, et al. C-reactive protein as a prognostic marker after lacunar stroke: levels of inflammatory markers in the treatment of stroke study. Stroke 2014;45:707-16.

13. Ryu JA, Yang JH, Lee D, et al. Clinical usefulness of procalcitonin and C-reactive protein as outcome predictors in critically ill patients with severe sepsis and septic shock. PLoS One 2015; 10:e0138150.

14. Windgassen EB, Funtowicz L, Lunsford TN, Harris LA, Mulvagh SL. C-reactive protein and high-sensitivity $C$-reactive protein: an update for clinicians. Postgrad Med 2011;123:114-9.

15. Hannan JL, Radwany SM, Albanese T. In-hospital mortality in patients older than 60 years with very low albumin levels. J Pain Symptom Manage 2012;43:631-7.

16. Sullivan DH, Roberson PK, Bopp MM. Hypoalbuminemia 3 months after hospital discharge: significance for long-term survival. J Am Geriatr Soc 2005;53:1222-6.

17. Kim MH, Ahn JY, Song JE, et al. The C-reactive protein/albumin ratio as an independent predictor of mortality in patients with severe sepsis or septic shock treated with early goal-directed therapy. PLoS One 2015;10:e0132109.

18. Xie Q, Zhou Y, Xu Z, et al. The ratio of CRP to prealbumin levels predict mortality in patients with hospital-acquired acute kidney injury. BMC Nephrol 2011;12:30.

19. World Health Organization. International statistical classification of diseases and related health problems 10th revision. 2nd ed. Geneva: World Health Organization, 2004.

20. Ranzani OT, Zampieri FG, Forte DN, Azevedo LC, Park M. C-reactive protein/albumin ratio predicts 90-day mortality of septic patients. PLoS One 2013;8:e59321.

21. Brabrand M, Folkestad L, Clausen NG, Knudsen T, Hallas J. Risk scoring systems for adults admitted to the emergency department: a systematic review. Scand J Trauma Resusc Emerg Med 2010;18:8.

22. Vincent JL, Moreno R, Takala J, et al. The SOFA (Sepsis-related Organ Failure Assessment) score to describe organ dysfunction/failure: on behalf of the Working Group on Sepsis-Related Problems of the European Society of Intensive Care Medicine. Intensive Care Med 1996;22:707-10. 